\title{
Investigating the role of temporal processing in developmental dyslexia: Evidence for a specific deficit in rapid visual segmentation
}

\author{
Luca Ronconi $^{1,2,3} \cdot$ David Melcher $^{3,4} \cdot$ Laura Franchin $^{4}$
}

Published online: 3 June 2020

(C) The Psychonomic Society, Inc. 2020

\begin{abstract}
The current study investigates the role of temporal processing in the visual domain in participants with developmental dyslexia (DD), the most common neurodevelopmental disorder, which is characterized by severe and specific difficulties in learning to read despite normal intelligence and adequate education. Specifically, our aim was to test whether DD is associated with a general impairment of temporal sensory processing or a specific deficit in temporal integration (which ensures stability of object identity and location) or segregation (which ensures sensitivity to changes in visual input). Participants with DD performed a task that measured both temporal integration and segregation using an identical sequence of two displays separated by a varying interstimulus interval (ISI) under two different task instructions. Results showed that participants with DD performed worse in the segregation task, with a shallower slope of the psychometric curve of percentage correct as a function of the ISI between the two target displays. Moreover, we found also a relationship between temporal segregation performance and text, words, and pseudowords reading speeds at the individual level. In contrast, no significant association between reading (dis)ability and temporal integration emerged. The current findings provide evidence for a difference in the fine temporal resolution of visual processing in DD and, considering the growing evidence about a link between visual temporal segregation and neural oscillations at specific frequencies, they support the idea that DD is characterized by an altered oscillatory sampling within the visual system.
\end{abstract}

Keywords Vision $\cdot$ Perception $\cdot$ Magnocellular-dorsal stream $\cdot$ Reading $\cdot$ Neural oscillations

A fundamental challenge for the brain is to parse continuous sensory input into meaningful units such as visual objects or spoken words. On the one hand, it is important to rapidly process and respond to changes in sensory input in order to notice change and guide action. However, the ability to integrate information over time is critical to accumulate evidence to make correct decisions based on more precise information.

Luca Ronconi

ronconi.luca@unisr.it

1 School of Psychology, Vita-Salute San Raffaele University, Via Olgettina 58, 20132 Milan, Italy

2 Division of Neuroscience, IRCCS San Raffaele Scientific Institute, Milan, Italy

3 Center for Mind/Brain Sciences (CIMeC), University of Trento, Rovereto, Italy

4 Department of Psychology and Cognitive Science, University of Trento, Rovereto, Italy
Thus, sensory processing involves a delicate balance between temporal integration and temporal segmentation.

Sensory processing has temporal resolution limits, ranging from a few milliseconds in auditory cortex ( $\mathrm{Lu}$, Liang, \& Wang, 2001) to tens of milliseconds in detection of visual flicker in early visual processing areas (Hawken, Shapley, \& Grosof, 1996; Hecht \& Shlaer, 1936). In terms of more complex processing routines, visual temporal resolution is on the order of $100 \mathrm{~ms}$ or more (Blake \& Lee, 2005; Pöppel, 2009; VanRullen, 2016; Wutz \& Melcher, 2014). One way to think of these temporal resolution limits is in terms of the time required to complete a specific perceptual, motor or cognitive computation cycle (Pöppel, 2009; VanRullen, 2016; Wutz \& Melcher, 2014), leading to the idea of temporal integration windows during which information is combined over time in order to process a packet of incoming sensory input. Any computation on sensory input, and in particular neural processing involving top-down and feedback loops, leads to discrete processing windows. Evidence has demonstrated the existence of such discrete (or quasidiscrete) temporal integration windows in visual (VanRullen, 2016; Wutz, Weisz, Braun, \& 
Melcher, 2014), auditory (Pöppel, 1997, 2009), and tactile (Fujisaki \& Nishida, 2009) processing and uncovered links to potential neural mechanisms (Ronconi, Oosterhof, Bonmassar, \& Melcher, 2017; van Wassenhove, 2016; VanRullen, 2016; Wutz, Melcher, \& Samaha, 2018).

Such temporal integration windows might play a role also in clinical differences in perceptual and cognitive tasks. For example, developmental dyslexia (DD) has been linked to a potential influence of temporal processing (Goswami, 2011; Goswami, Power, Lallier\& Facoetti, 2014). This common neurodevelopmental disorder, thought to represent around $80 \%$ of all specific learning disorders, describes a severe and specific difficulty in learning to read even in persons with normal intelligence and education experience. Around 10\% of children, across cultures, have this difficulty in learning to read, which then creates problems in their education and challenges for everyday life (Shaywitz et al., 2004). Since both pure linguistic and more perceptual deficits have been repeatedly found in DD, this raises the question of whether there might be a core sensory processing component that could underlie both issues (Goswami et al., 2014).

A number of studies have demonstrated differences in temporal processing between participants with DD and controls. These involve both auditory (Goswami et al., 2002; Hornickel \& Kraus, 2013; Tallal, 1980) and visual tasks (Boets, Vandermosten, Cornelissen, Wouters, \& Ghesquière, 2011; Galaburda \& Livingstone, 1993; Gori et al., 2015; Gori et al., 2016; Kevan \& Pammer, 2008, 2009; Menghini et al., 2010; Stein \& Talcott, 1999; Tallal, 2004), as well as spatiotemporal aspects of attention (Bosse, Tainturier, \& Valdois, 2007; Facoetti et al., 2010a, b; Hari \& Renvall, 2001; Roach \& Hogben, 2007; Visser, Boden, \& Giaschi, 2004). This complex pattern of findings of differences, as well as failures to find differences (Johannes, Kussmaul, Münte, \& Mangun, 1996), has led to a series of theories regarding the underlying mechanisms of DD, in terms of neural substrates and computational underpinnings (Goswami, 2011; Menghini et al., 2010; Stein, 2019; Stein \& Walsh, 1997), with at least a dozen competing theories (Ramus \& Ahissar, 2012).

One challenge in understanding the role of temporal factors in DD, or indeed in any potential difference between groups based on age or clinical diagnosis, is to control for other confounding variables that might be involved in the task. In a temporal simultaneity or motion processing task, for example, it might be difficult to distinguish between specific temporal processing differences and more general performance factors, such as sustained attention, response bias, motivation, or motor errors.

To address this issue, we used a variant of the missing element task (Di Lollo, 1980). Specifically, in this segregation/integration ("SegInt") task (Freschl, Melcher, Kaldy, \& Blaser, 2019; Ronconi, Busch, \& Melcher, 2018; Sharp, Melcher, \& Hickey, 2018, 2019; Wutz et al., 2018;
Wultz, Muschter, van Koningsbruggen, Weisz, \& Melcher, 2016) participants see two displays separated by a varying interstimulus interval (ISI). Each of the two displays contains seven full annuli, placed in random locations, and an "odd element" with a half annulus, such that the two half annuli complemented each other across displays in the same position. Finding the position of the single odd element requires segregating the displays over time to avoid confusing it with the other full annuli. Finding the position of the single empty location requires instead integrating the displays over time (see Fig. 1).

The use of both a segregation and an integration task with the same participants and stimuli allowed us to distinguish between two possible mechanisms that might underlie group differences. The first potential mechanism, as described above, is an enlarged temporal integration window in dyslexia. An enlarged integration window would temporally smear both visual and auditory input, which would result in reduced sensitivity to the minute temporal fluctuations due to less temporal resolution in perceptual processing. Such a mechanism would result in a specific pattern in which the crossover point would shift to the right in the psychometric curve (see Fig. 1a): A reduced performance as a function of time with the segmentation task would be accompanied by better performance in the integration task. Another possible mechanism, however, would be a specific difficulty in the rapid segmentation task for DD participants. Studies have shown evidence for top-down control of temporal resolution in visual (Ronconi, Pincham, Cristoforetti, Facoetti, and Szucs, 2016a; Ronconi, Pincham, Szucs, and Facoetti, 2016b; Samaha, Bauer, Cimaroli, \& Postle, 2015; Wutz et al., 2018) and auditory (Zion Golumbic, Poeppel, \& Schroeder, 2012) tasks. These experiments suggest that people are able to fine-tune their temporal rhythms to enhance performance in processing rapid visual input and spoken language. Reduced top-down control over visual temporal resolution would result in a shallower slope for segmentation trials (see Fig. 1b), since DD participants would need more time to achieve the same performance as controls, or else the segmentation curve could be shifted rightward or downward (see Fig. 1c), with no effect on the integration curve, if segmentation is generally "harder" for these participants.

There is some inconsistency in past works studying temporal integration or rapid segregation tasks for children with DD (Arnett \& Di Lollo, 1979; Di Lollo, Hanson, \& McIntyre, 1983; Fisher \& Frankfurter, 1977; Stanley \& Hall, 1973). Some studies have suggested that poor readers also performed worse in tasks requiring rapid processing (Di Lollo et al., 1983; Facoetti, Ruffino, Peru, Paganoni, \& Chelazzi, 2008; Stanley \& Hall, 1973), while others have argued for no difference when controlling for age (Arnett \& Di Lollo, 1979) or 


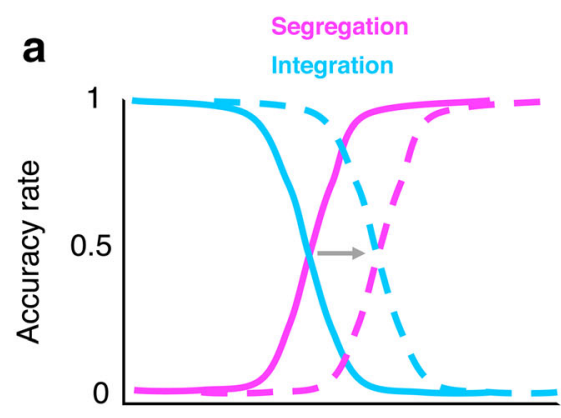

ISI

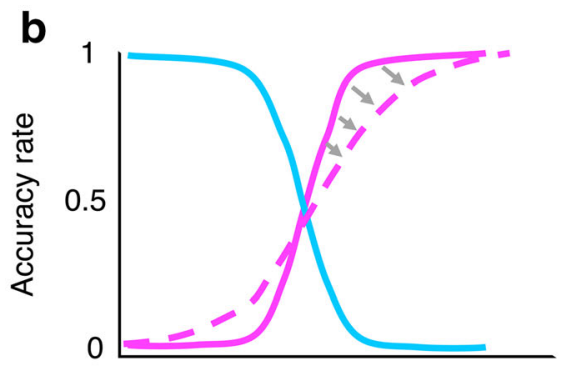

$|S|$

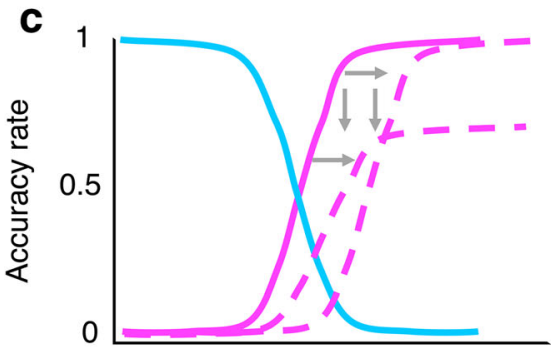

ISI
General rightward shift

Longer temporal integration window

\section{Shallow slope in segregation Low top-down control}

Rightward/downward shift of the segregation curve General reduction in temporal segregation (i.e. difficulty with temporal resolution)

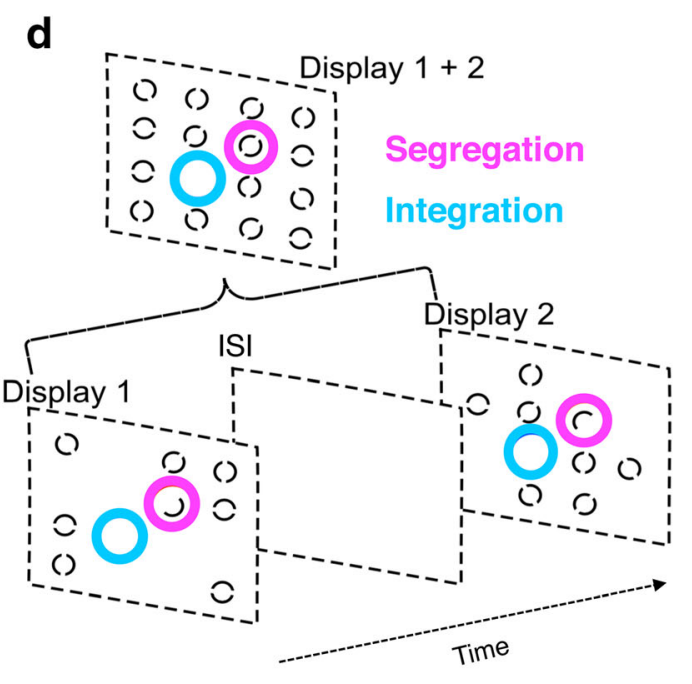

Fig. 1 Schematic representation of the hypotheses concerning temporal processing in DD (a-c) and schematic representation of the task procedure (d) used to evaluate temporal integration and segregation in the present study (ISI = interstimulus interval)

even for faster processing in participants with DD in a task using backward masking of letters (Fisher \& Frankfurter, 1977). However, the latter study presented items for $200 \mathrm{~ms}$ prior to masking, making it insensitive to the type of rapid visual segregation tasks usually used to study processing on the order of $100 \mathrm{~ms}$. The overall pattern of results has lead Di Lollo et al. (1983) to suggest that the "dyslexic visual system may take an unusually long period of time to recover from the aftereffects of neural activity evoked by an inducing stimulus" (p. 923).

To test whether there might be a general temporal resolution difference in DD, we recruited a sample of adults diagnosed with DD to measure performance in the SegInt task. Our aim was to distinguish between a general shift toward longer/slower sensory processing or a specific deficit in rapid temporal segregation. In addition, by also testing reading in the control group, we were able to look at correlations at the individual participant level between reading and performance in the task, which might be present even in individuals without a DD diagnosis.

\section{Method}

\section{Participants}

A total of 41 adults were recruited for this study (age range: $18-41$ years; mean age $=29.5$ years $), 20$ adults $(13$ males and seven females) with DD and 21 (11 males and nine females) control participants with normal reading abilities. All participants were native Italian speakers without a history of brain damage, language problems, or psychiatric symptoms, and all observers reported normal or corrected-to-normal vision and hearing. Participants' level of education ranged between 13 and 15 years. The majority of them $(n=29)$ were college students enrolled at the University of Trento (Italy); five were high school students and seven had finished education and were currently employed.

Participants with DD were recruited through the University Office for Student With Special Needs and by advertisement. All of them possessed an official diagnosis completed by registered clinical psychologists at the University or Mental 
Health Services. They were paid (€8) for their participation. Adults with DD were contrasted with a control group, which consisted of adults with normal reading abilities and no history of reading difficulties. The control group was recruited through class announcements and received university credits. The research project was approved by the Ethical Committee of the University of Trento, and all participants gave their informed consent.

All participants completed a battery of tests to provide a better understanding of the cognitive and reading skills of each group. Group characteristics are presented in Table 1 . In detail, the battery contained the following:

Intelligence score An estimation of the general cognitive level was measured with the Raven's Advanced Progressive Matrices Test (Raven, 1998). This measure required the participants to make judgments related to presented problems and to indicate their choice by pointing to the correct answer. The raw score was calculated as the number correctly identified items for a maximum achievable score of 60 .

Text reading score It was assessed through the use of a text entitled "Funghi in città" (written by Italo Calvino, 1993, in "Marcovaldo," as reported and standardized in Judica \& De Luca, 1993). This text is characterized by a significant grammatical, lexical, and syntactical complexity that allows to better individuate the different characteristics in reading when testing adult participants. Performance was measured in respect to speed and accuracy of reading. The speed score was calculated by dividing the time (in seconds) taken to read the complete text by the number of read syllables $(n=571)$. The accuracy score was calculated as the number of mistakes made (for example, 1 for inversion errors and 0.5 for accent shift errors).

Words and pseudowords reading This was assessed by asking participants to read lists of single words and pseudowords (taken from Sartori, Job, \& Tressoldi, 1995). Specifically, the participants' task was to read four lists of word and three lists of nonword. Their performance was measured with respect to speed and accuracy of reading. The speed score was calculated as the time taken to read one list at a time, and the accuracy score was calculated in the same way as text reading test.

\section{Apparatus and stimuli}

All visual stimuli were displayed on a 22.5-in. VIEWPixx monitor with a vertical refresh rate of $100 \mathrm{~Hz}$. The stimulus for the main task was made up of two displays that were shown sequentially, separated by a blank interstimulus interval (ISI; see Fig. 1d). The two target displays contained annuli placed within an invisible $4 \times 4$ quadratic element grid (each square was $1 \times 1$ degree). These stimuli were shown in two different and separated frames. Seven random locations (14 over both frames out of 16 total) were filled with a full black annulus on a uniform gray background (0.5-degree size, 0.06 degree line width; 0.5 -degree space between grid locations). Each annulus was split by a central gap with a randomly chosen orientation that could be $0^{\circ}, 45^{\circ}, 90^{\circ}$, or $135^{\circ}$. In addition, there was one "odd element" with a half annulus in each of the two displays, such that the two half annuli complemented each other across displays. Finally, one location was left empty. The stimuli were similar to those used in previous studies with the segregation/integration paradigm (Freschl et al., 2019; Ronconi et al., 2018; Sharp et al., 2018, 2019; Wutz et al., 2018; Wutz et al., 2016). The experiment was programmed in MATLAB, using Psychtoolbox (Brainard, 1997), and all visual stimuli were displayed on a middle grey background.

\section{Procedure}

Each target display (containing the seven circles and one halfcircle) was shown for one refresh cycle $(10 \mathrm{~ms})$ separated by

Table 1 Descriptive statistics of participants with DD and typical readers (controls) for each cognitive and reading measure

\begin{tabular}{llll}
\hline & $\begin{array}{l}\text { Controls }(N=21) \\
\text { Mean }(S D)\end{array}$ & $\begin{array}{l}\text { DD }(N=20) \\
\text { Mean }(S D)\end{array}$ & $p$ value $(t$ test $)$ \\
\hline Raven's APM score & $8.33(2.4)$ & $6.95(2.6)$ & .081 \\
Text reading speed & $0.74(1.1)$ & $-4.92(3.8)$ & $<.001$ \\
Text reading accuracy & $0.55(0.8)$ & $-2.91(3.1)$ & $<.001$ \\
Words reading speed & $0.43(1.0)$ & $-2.36(2.8)$ & $<.001$ \\
Words reading accuracy & $0.33(0.6)$ & $-1.19(2.0)$ & .004 \\
Pseudowords reading speed & $0.51(0.7)$ & $-2.41(2.3)$ & .001 \\
Pseudowords reading accuracy & $0.65(0.6)$ & $-0.48(1.7)$ & .009
\end{tabular}

Note. All reading measures are reported in $z$ scores; $t$ and $p$ values were derived from independent-samples $t$ tests. APM $=$ Advanced Progressive Matrices 
one of 10 possible ISI durations (i.e., blank screen) that ranged between 10 and $100 \mathrm{~ms}$ in steps of $10 \mathrm{~ms}$ (see Fig. 1d). These values for the ISI were chosen based on our previous studies (Freschl et al., 2019; Ronconi et al., 2018; Sharp et al., 2018, 2019; Wutz et al., 2018; Wutz et al., 2016) to allow us to find both good (near $100 \%$ ) and poor (near chance) performance on both tasks, with integration performance best for short values of ISI and worst for long ISIs, and the opposite trend with temporal segregation.

In different blocks, participants were instructed to localize either the odd element (segregation task) or the empty location (integration task; see Fig. 1d). Crucially, finding the odd element requires segregating the displays over time, whereas integration results in the perception of a full annulus, identical to the 14 other annuli. In contrast, finding the missing element (i.e., the empty location) in both displays requires integrating the displays over time, whereas segregation results in the perception of two separate displays with many empty locations. Both the odd and missing elements were shown in all trials, so that integration and segregation were tested using the identical stimuli, only using different task instructions. At the end of each trial, participants were asked to report the correct location by clicking on the corresponding stimulus position in the grid, reporting the location of the odd element (half circle) in the segregation trials, or the location of the missing element in the integration trials. The response was given with no time constraints. The total amount of trials administered for each participant were 540, consisting of 270 segregation trials and 270 integration trials (27 for each ISI value).

\section{Results}

\section{Temporal integration and segregation performance in $\mathrm{DD}$ versus controls}

As expected, integration and segregation performance depended on the ISI (see Fig. 2a-b). We first examined our hypotheses by means of an analysis of variance (ANOVA) on accuracy scores, with condition (segregation vs. integration) and ISI (10 levels) used as within-subjects factors, and group (DD vs. controls) as the between-subjects factor. The ANOVA revealed a significant main effect of condition, $F(1,39)=9.848, p<.003$, showing that the task completed in each block of trials (segregation vs. integration) influenced performance. There was also a significant main effect of ISI, $F(9,351)=22.737, p<.001$, and an interaction between condition and ISI, $F(9,351)=422.041, p<.001$, was found. This confirmed the general trend found in previous studies in which accuracy increased with longer ISIs for the segregation task, but decreased as a function of ISI in the integration task (Freschl et al., 2019; Sharp et al., 2018, 2019; Wutz et al., 2016). Importantly, a main effect of group emerged, $F(1$,
$39)=5.302, p<.027$, revealing that participants with $\mathrm{DD}$ performed the task with lower accuracy (mean $=0.594$, SEM $=0.024$ ) relative to controls (mean $=0.659, S E M=0.015$ ).

To further explore the significant main effect of group, we performed a series of independent-samples $t$ tests to investigate whether specific ISIs were associated with significantly different scores. Only for the segregation condition, we found that accuracy at ISI $=50 \mathrm{~ms}, t(39)=2.06, p=.046), \mathrm{ISI}=70$ $\mathrm{ms}, t(39)=2.08, p=.044$, and ISI $=100 \mathrm{~ms}, t(39)=2.23, p=$ .032 , were significantly different between participants with DD and controls. In the group data, it is clear that segregation performance reached 75\% performance much earlier (around $40 \mathrm{~ms}$ ) for the control group compared to the DD group, and performance in the latter group remained lower even at the longest ISI tested $(100 \mathrm{~ms})$.

In the second analysis used to test our hypothesis, for each participant we fit the accuracy data as a function of ISI with a psychometric curve (logistic) to extract the 50\% and $75 \%$ thresholds and slope values, separately for segregation and integration (see Fig. 2c-d). This allowed us to test whether segregation performance increased more quickly as a function of ISI in one of the two groups. In segregation trials, there was indeed a significant difference in the slope of the psychometric curve between the two groups, $t(32.86)=-2.726, p=.010$. The difference in the $50 \%$ threshold was not significant, $t(21.58)=-1.302, p=.207$, and the difference in the $75 \%$ threshold was more pronounced, but did not reach significance in a two-tailed test, $t(39)=1.84, p=.074$. In terms of individual scores, $35 \%$ of participants with DD exhibited slope values that were below one standard deviation relative to the distribution calculated in the control group, and $70 \%$ scored below 0.5 standard deviations. In integration trials, no significant differences between DD and control group emerged for either slope or threshold (both $p \mathrm{~s}>.16$ ).

\section{Correlations between temporal integration/segregation and reading skills}

Next, we tested whether individuals who had more difficulty with the segregation task were also those with lower reading scores. To do this, we measured the correlation (Pearson) between temporal segregation and the three measures of reading skills (text reading, word reading, and pseudoword reading) across the entire sample of participants. We found that the individual slope values correlated positively with the speed in text reading ( $r=.355, p=.023$; Fig. 3a), in word reading $(r=.344, p=.028$; Fig. $3 \mathrm{~b})$, and in pseudoword reading $(r=$ $.368, p=.018$; Fig. $3 c$ ), confirming at an individual participant level the specific relationship between temporal segregation and reading skills that emerged in the group analyses. Participants who showed the fastest improvement in segregation as a function of ISI had higher reading scores. 

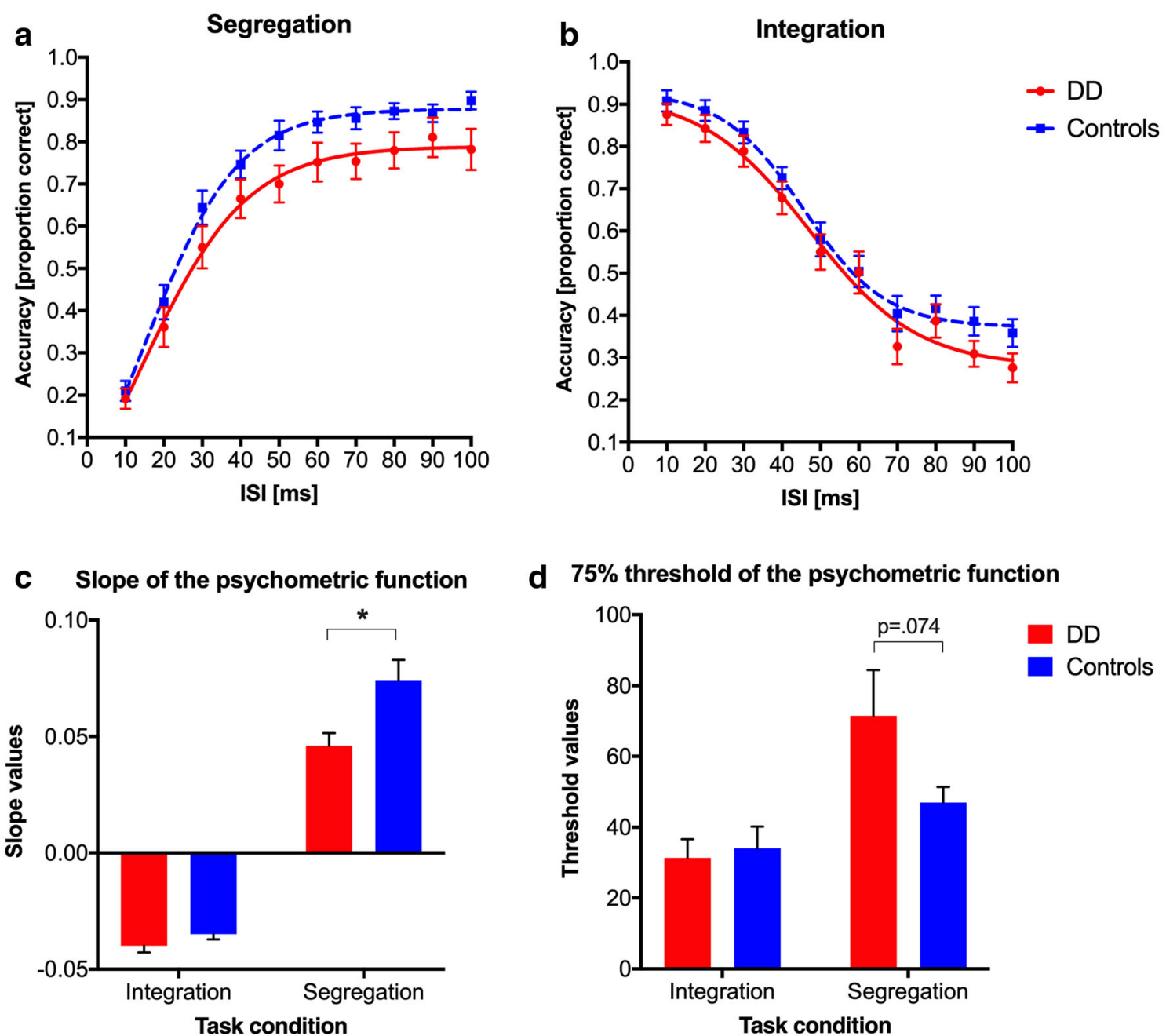

d $75 \%$ threshold of the psychometric function

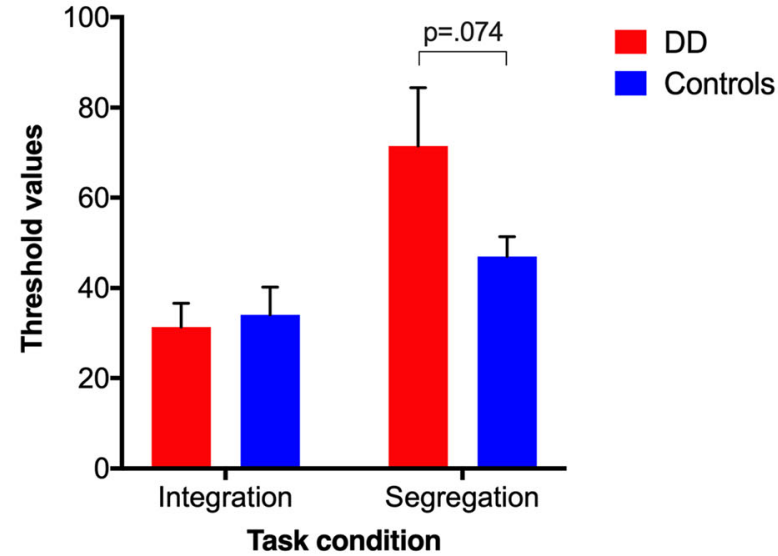

Fig. 2 Average accuracy rates in the two experimental conditions, (a) segregation and (b) integration, as a function of interstimulus interval (ISI) and group (developmental dyslexia = DD vs. controls). The psychometric curves plotted here were fitted on the aggregated data for

illustration purposes. Average slope (c) and 75\% threshold values (d) obtained from fitting a psychometric (logistic) function to the individual data. Error bars show the SEM. * $p<.05$

When correlations were performed separately within the two groups, no significant correlations emerged between segregation slopes and reading scores. Interestingly, control

participants with typical reading skills showed a significant negative correlation between segregation threshold and text reading accuracy $(r=-.524, p=.015)$, perhaps reflecting a
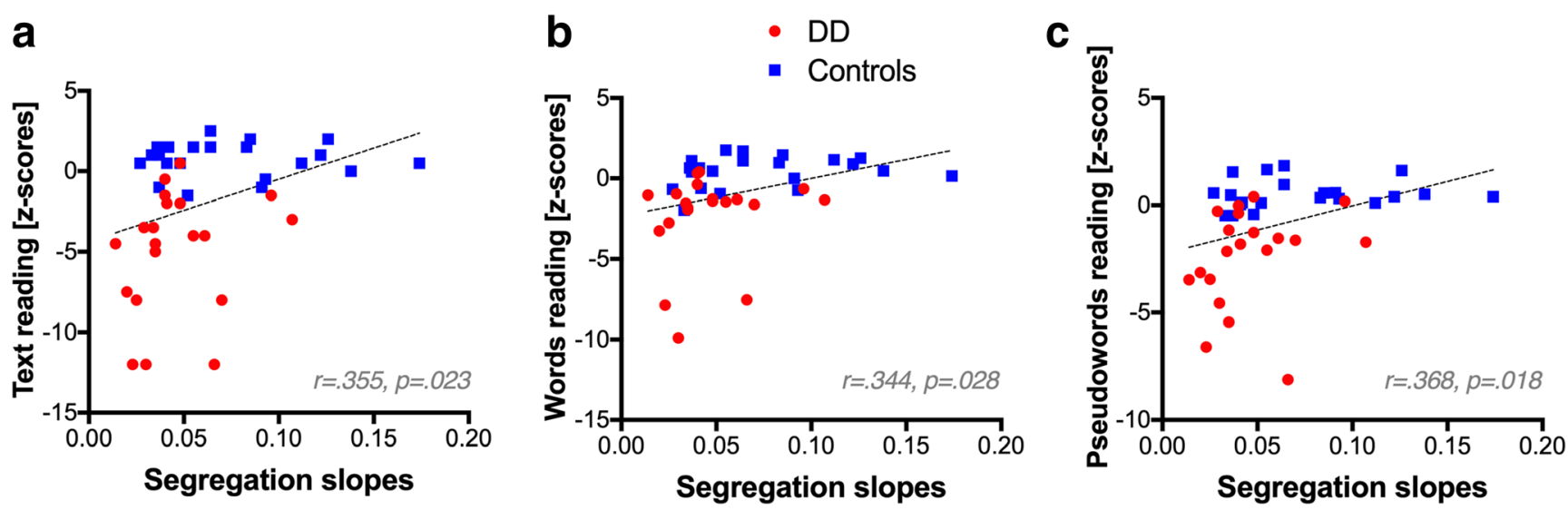

Fig. 3 Correlation between the slope values of the psychometric function for the segregation condition and the speed ( $z$ scores) in text reading (a), word reading $(\mathbf{b})$, and pseudoword reading $(\mathbf{c})$ 
speed-accuracy trade-off, while in the sample of participants with DD, no correlations between reading scores and segregation threshold resulted significant.

\section{Discussion}

The current findings point to a specific difference in the ability to segregate two rapidly presented sequential visual inputs in participants with DD compared with a control group. The DD participants performed worse in the segregation task, with a significantly shallower slope of the psychometric curve (percentage correct as a function of ISI). In other words, the temporal resolution was less precise in the DD group. There was no significant difference between groups in performance in the task requiring temporal integration. These results confirm and extend previous work that has investigated DD participants in the missing-dot task (temporal integration) or with tasks involving rapid presentation of items (temporal segregation). Unlike previous studies with motion processing (for a review, see Stein, 2019), which involves both rapid processing and also spatiotemporal integration (in order to bind two subsequent images into a percept of motion), we found a specific deficit in temporal segregation and no difference in the integration task. Moreover, we also found a relationship between temporal segregation and reading performance at the individual level, whereby individuals who had more difficulty with the segregation task were also those with lower reading speed in text, words, and pseudowords.

Together, this pattern of results is consistent with the idea that DD reflects a more general temporal processing difference that is not limited to the linguistic stimuli or to processing in the auditory modality (Facoetti et al., 2008; Facoetti et al., 2010b; McLean, Stuart, Coltheart, \& Castles, 2011; Stanley \& Hall, 1973). Our findings can be considered within the different theoretical frameworks that have been brought to bear on the question of how and why there might be such temporal processing differences and their potential role in DD and other developmental disorders. As reviewed in the Introduction, there are currently at least a dozen competing theories of $\mathrm{DD}$, several of which involve temporal processing, so we will focus on those most relevant to the question of a role for sensory or perceptual mechanisms.

The magnocellular-dorsal (MD) theory of DD has been developed based on a number of studies showing impairment of DD children in some motion-processing tasks and other tasks related to the function of visual MD pathway (Gori \& Facoetti, 2015; Livingstone, Rosen, Drislane, \& Galaburda, 1991; Stein, 2019; Stein \& Walsh, 1997; Vidyasagar \& Pammer, 1999, 2010; Visser et al., 2004). The magnocellular pathway describes the passing of information from retinal ganglion cells through the $\mathrm{M}$ layer of the lateral geniculate nucleus, which then reaches visual processing areas in occipital and parietal cortices (Maunsell \& Newsome, 1987). Neurons in this pathway respond well to luminance contrast, low spatial frequencies, and high temporal frequencies, and are linked to processing of both real and illusory motion stimuli (Livingstone \& Hubel, 1987; Morrone et al., 2000). A role for the MD pathway in rapid temporal processing is suggested by a study showing differences in flicker thresholds for magnocellular-targeted stimuli in children with dyslexia (McLean et al., 2011). At the same time, however, the role of the MD pathway has not been specifically tested with the SegInt stimulus. One possibility is that the segregation task might make use of transient responses to the onset and offset of the stimulus, which should be better processed in the MD pathway than in the slower parvocellular pathway. Moreover, estimates of temporal integration in contrast threshold tasks suggest a window of around $40-50 \mathrm{~ms}$ for the MD pathway (Pokorny, 2011), which corresponds to the crossover point in the SegInt task.

Our results can also fit within the temporal sampling framework theory of DD (Goswami, 2011). In terms of auditory processing, the idea is that dyslexia reflects a deficit in the entraining of neural oscillations to the temporal envelope of the different speech features (phonemes, syllables, words). Although that theory initially focused on temporal sampling windows in spoken language, more recently it has been broadened to consider deficits in visual and audio-visual processing (Goswami et al., 2014; Pammer, 2014). Pammer (2014), for example, argued that "as in the auditory domain, temporal coding here really refers to the ability of the visual system to effectively process quickly presented information . . . both processing speed and the ability of the visual system to deal with information presented quickly, such as at the rate of saccadic and fixation sequences, or even faster" (p. 6). Indeed, there is recent evidence for a link between visual temporal integration/segregation and neural oscillations in the alpha $(8-12 \mathrm{~Hz})$ and theta $(4-7 \mathrm{~Hz})$ band (Ronconi et al., 2018; Ronconi \& Melcher, 2017; Ronconi et al., 2017; Ronconi, Pincham, Cristoforetti, et al., 2016a; Samaha \& Postle, 2015; Wutz et al., 2018; Wutz et al., 2016), which is quite similar to the link between language processing and oscillations. Although the link between theta/alpha oscillations in vision and reading (dis)abilities has been advanced at a theoretical level (Vidyasagar, 2013, 2019), at present, direct neurophysiological investigations are needed to clarify whether the segregation deficit report here could be linked to dysfunctional oscillatory activity in the theta/alpha band within the visual system.

As described in the Introduction, our pattern of results could also be interpreted in terms of a reduced ability to fine-tune the temporal sampling process in order to "speed up" the temporal resolution of visual processing. This interpretation of the results would also fit with the temporal sampling framework since both reading and spoken language 
processing involve the ability to entrain sensory processing rhythms to the temporal envelope of the sensory signal, and there is evidence for a deficit in this type of entrainment to auditory stimuli (Goswami, 2011; Pammer, 2014). In future work, it would be useful to further investigate whether reduced performance in rapid visual presentation tasks is due to a more sluggish visual resolution, to a less flexible topdown control over temporal resolution, or both (see Pammer, 2014, for a similar argument).

Another set of studies point to a role of spatial (or spatiotemporal) attention in dyslexia (Bosse et al., 2007; Facoetti et al., 2008; Menghini et al., 2010; Roach \& Hogben, 2007; Visser et al., 2004). In recent studies, the influence of both spatial and temporal attention on performance in the SegInt task revealed that spatial attention benefitted performance in both the integration and the segregation task (Sharp et al., 2018, 2019). Therefore, any deficit in spatial attention in DD participants should have been visible in both tasks, rather than specific to rapid segregation as we found here. A more specific scenario is predicted by the sluggish attentional shifting (SAS) theory (Hari \& Renvall, 2001). This theory predicts that when DD individuals process rapid stimulus sequences, their attention cannot disengage fast enough to move from one item to the next. In line with the SAS, Lallier and colleagues (Lallier et al., 2010; Lallier et al., 2009) used a stream segregation task and showed that participants with DD required more time to rapidly segregate two visual streams appearing in different spatial positions. However, in the SegInt task segregation is accomplished without the need to move spatial attention from one location to another. Moreover, in the previous study testing the effect of temporal attention in the SegInt task, there was an invalid cue cost in temporal integration but no effect on rapid segregation (Sharp et al., 2019). Thus, the overall pattern of results from this study is not consistent with our effect being caused by a core deficit in spatial or spatiotemporal attention. Conversely, some previous studies showing temporal attention effects might have been influenced by difficulties in rapid temporal segmentation. The reduced ability to move from one item to the next (Hari \& Renvall, 2001; Krause, 2015) might actually result, at least in part, from slower temporal resolution in completing the perceptual processing of each item during rapid visual presentation (RSVP) of items (Goswami et al., 2014). Interestingly, the RSVP sequence timing used in many studies has been quite similar to that of the temporal segregation task used here (e.g., Visser et al., 2004). Participants with reduced temporal resolution might be expected to dwell longer on targets in order to process them and to be less able to rapidly shift processing to subsequent items (see Pammer, 2014, for a similar argument).

In conclusion, the current findings provide further evidence for a difference in the temporal resolution of visual processing in DD. In the context of the existing literature, our results support the idea that temporal processing differences in early age, whether auditory, visual or multimodal, may make learning to read more difficult. In future work, it would be valuable to see whether such differences are amenable to training, such that children with an early diagnosis of DD might be able to learn to "speed up" their visual processing or fine-tune their neural sampling rhythms to better match the pattern of sensory input. As an example, action video game (AVG) training can improve performance in tasks such as the attentional blink and backward masking, which also entail temporal segregation mechanisms (Bavelier \& Green, 2019). AVG training has also been shown to improve reading efficiency in children with DD (Bertoni, Franceschini, Ronconi, Gori, \& Facoetti, 2019; Franceschini et al., 2013; Franceschini et al., 2017; Gori et al., 2016). Future investigations might test temporal segregation as a possible mediator of the effect of AVG on reading skills. Given that visual temporal resolution varies between individuals (Samaha \& Postle, 2015), across tasks (Ronconi et al., 2017) and across the life span (Arnett \& Di Lollo, 1979; Di Lollo, Arnett, \& Kruk, 1982; Dobkins, Anderson, \& Lia, 1999), it is also possible that genetically linked features (Mascheretti et al., 2018) interact with training and environmental factors.

Acknowledgements We would like to thank Isabel Casagrande for her help in the data collection. This research was supported by a European Research Council grant "Construction of Perceptual Space-Time" (StG Agreement 313658) awarded to D.M.

Open practices statement The data and materials for this study are available upon request to the authors.

\section{Compliance with ethical standards}

Conflict of interest The authors declare no competing interests.

\section{References}

Arnett, J. L., \& Di Lollo, V. (1979). Visual information processing in relation to age and to reading ability. Journal of Experimental Child Psychology, 27(1), 143-152. doi:https://doi.org/10.1016/00220965(79)90066-3

Bavelier, D., \& Green, C. S. (2019). Enhancing attentional control: Lessons from action video games. Neuron, 104(1), 147-163. doi: https://doi.org/10.1016/j.neuron.2019.09.031

Bertoni, S., Franceschini, S., Ronconi, L., Gori, S., \& Facoetti, A. (2019). Is excessive visual crowding causally linked to developmental dyslexia? Neuropsychologia, 130, 107-117. doi:https://doi.org/10. 1016/j.neuropsychologia.2019.04.018

Blake, R., \& Lee, S.-H. (2005). The role of temporal structure in human vision. Behavioral and Cognitive Neuroscience Reviews, 4(1), $21-$ 42. doi:https://doi.org/10.1177/1534582305276839

Boets, B., Vandermosten, M., Cornelissen, P., Wouters, J., \& Ghesquière, P. (2011). Coherent motion sensitivity and reading development in the transition from prereading to reading stage. Child Development, 82(3), 854-869. doi:https://doi.org/10.1111/j.1467-8624.2010. 01527.x 
Bosse, M.-L., Tainturier, M. J., \& Valdois, S. (2007). Developmental dyslexia: The visual attention span deficit hypothesis. Cognition, 104(2), 198-230.

Brainard, D. H. (1997). The Psychophysics Toolbox. Spatial Vision, 10(4), 433-436.

Di Lollo, V. (1980). Temporal integration in visual memory. Journal of Experimental Psychology: General, 109(1), 75-97. doi:https://doi. org/10.1037/0096-3445.109.1.75

Di Lollo, V., Arnett, J. L., \& Kruk, R. V. (1982). Age-related changes in rate of visual information processing. Journal of Experimental Psychology: Human Perception and Performance, 8(2), 225-237. doi:https://doi.org/10.1037/0096-1523.8.2.225

Di Lollo, V., Hanson, D., \& McIntyre, J. S. (1983). Initial stages of visual information processing in dyslexia. Journal of Experimental Psychology: Human Perception and Performance, 9(6), 923-935. doi:https://doi.org/10.1037/0096-1523.9.6.923

Dobkins, K. R., Anderson, C. M., \& Lia, B. (1999). Infant temporal contrast sensitivity functions (tCSFs) mature earlier for luminance than for chromatic stimuli: Evidence for precocious magnocellular development? Vision Research, 39(19), 3223-3239. doi:https://doi. org/10.1016/S0042-6989(99)00020-6

Facoetti, A., Corradi, N., Ruffino, M., Gori, S., \& Zorzi, M. (2010a). Visual spatial attention and speech segmentation are both impaired in preschoolers at familial risk for developmental dyslexia. Dyslexia, 16(3), 226-239.

Facoetti, A, Ruffino, M., Peru, A., Paganoni, P., \& Chelazzi, L. (2008). Sluggish engagement and disengagement of non-spatial attention in dyslexic children. Cortex, 44(9), 1221-1233.

Facoetti, A., Trussardi, A. N., Ruffino, M., Lorusso, M. L., Cattaneo, C., Galli, R., ... Zorzi, M. (2010b). Multisensory spatial attention deficits are predictive of phonological decoding skills in developmental dyslexia. Journal of Cognitive Neuroscience, 22(5), 1011-1025.

Fisher, D. F., \& Frankfurter, A. (1977). Normal and disabled readers can locate and identify letters: Where's the perceptual deficit? Journal of Reading Behavior, 9(1), 31-43. doi:https://doi.org/10.1080/ 10862967709547205

Franceschini, S., Gori, S., Ruffino, M., Viola, S., Molteni, M., \& Facoetti, A. (2013). Action video games make dyslexic children read better. Current Biology, 23(6), 462-466. https://doi.org/10.1016/j.cub. 2013.01.044

Franceschini, Sandro, Trevisan, P., Ronconi, L., Bertoni, S., Colmar, S., Double, K., Facoetti, A., \& Gori, S. (2017). Action video games improve reading abilities and visual-to-auditory attentional shifting in English-speaking children with dyslexia. Scientific Reports, 7(1), 5863. doi:https://doi.org/10.1038/s41598-017-05826-8

Freschl, J., Melcher, D., Kaldy, Z., \& Blaser, E. (2019). Visual temporal integration windows are adult-like in 5- to 7-year-old children. Journal of Vision, 19(7), 5. doi:https://doi.org/10.1167/19.7.5

Fujisaki, W., \& Nishida, S. (2009). Audio-tactile superiority over visuotactile and audio-visual combinations in the temporal resolution of synchrony perception. Experimental Brain Research, 198(2/3), 245-259. doi:https://doi.org/10.1007/s00221-009-1870-x

Galaburda, A., \& Livingstone, M. (1993). Evidence for a magnocellular defect in developmental dyslexia. Annals of the New York Academy of Sciences, 682(1), 70-82. doi:https://doi.org/10.1111/j.1749-6632. 1993.tb22960.x

Gori, S., \& Facoetti, A. (2015). How the visual aspects can be crucial in reading acquisition? The intriguing case of crowding and developmental dyslexia. Journal of Vision, 15(1), 8-8. doi:https://doi.org/ $10.1167 / 15.1 .8$

Gori, S., Mascheretti, S., Giora, E., Ronconi, L., Ruffino, M., Quadrelli, E., Facoetti, A., \& Marino, C. (2015). The DCDC2 Intron 2 Deletion impairs illusory motion perception unveiling the selective role of magnocellular-dorsal stream in reading (dis)ability. Cerebral Cortex, 25(6), 1685-1695. doi:https://doi.org/10.1093/cercor/ bhu 234
Gori, S., Seitz, A. R., Ronconi, L., Franceschini, S., \& Facoetti, A. (2016). Multiple causal links between magnocellular-dorsal pathway deficit and developmental dyslexia. Cerebral Cortex, 26(11), 4356-4369.

Goswami, U. (2011). A temporal sampling framework for developmental dyslexia. Trends in Cognitive Sciences, 15(1), 3-10.

Goswami, U., Power, A. J., Lallier, M., \& Facoetti, A. (2014). Oscillatory "temporal sampling" and developmental dyslexia: Toward an overarching theoretical framework. Frontiers in Human Neuroscience, 8(November), 904. doi:https://doi.org/10.3389/fnhum.2014.00904

Goswami, U., Thomson, J., Richardson, U., Stainthorp, R., Hughes, D., Rosen, S., \& Scott, S. K. (2002). Amplitude envelope onsets and developmental dyslexia: A new hypothesis. Proceedings of the National Academy of Sciences, 99(16), 10911-10916. doi:https:// doi.org/10.1073/PNAS.122368599

Hari, R., \& Renvall, H. (2001). Impaired processing of rapid stimulus sequences in dyslexia. Trends in Cognitive Sciences, 5(12), 525532.

Hawken, M. J., Shapley, R. M., \& Grosof, D. H. (1996). Temporalfrequency selectivity in monkey visual cortex. Visual Neuroscience, 13(3), 477-492. doi:https://doi.org/10.1017/ s0952523800008154

Hecht, S., \& Shlaer, S. (1936). intermittent stimulation by light: V. The relation between intensity and critical frequency for different parts of the spectrum. The Journal of General Physiology, 19(6), 965-977. doi:https://doi.org/10.1085/jgp.19.6.965

Hornickel, J., \& Kraus, N. (2013). Unstable representation of sound: A biological marker of dyslexia. Journal of Neuroscience, 33(8), 3500-3504. doi:https://doi.org/10.1523/JNEUROSCI.4205-12. 2013

Johannes, S., Kussmaul, C. L., Münte, T. F., \& Mangun, G. R. (1996). Developmental dyslexia: Passive visual stimulation provides no evidence for a magnocellular processing defect. Neuropsychologia, 34(11), 1123-1127. doi:https://doi.org/10.1016/0028-3932(96) 00026-7

Judica, A., \& De Luca, M. (1993). Prova di velocità di lettura brani per la scuola media superiore [Speed reading test for high school]. Retrieved from https://www.hsantalucia.it/sites/default/files/fsl_ labdislessia lettura brani test.pdf

Kevan, A., \& Pammer, K. (2008). Visual deficits in pre-readers at familial risk for dyslexia. Vision Research, 48(28), 2835-2839.

Kevan, A., \& Pammer, K. (2009). Predicting early reading skills from pre-reading measures of dorsal stream functioning. Neuropsychologia, 47(14), 3174-3181.

Krause, M. B. (2015). Pay attention!: Sluggish multisensory attentional shifting as a core deficit in developmental dyslexia. Dyslexia, 21(4), 285-303. doi:https://doi.org/10.1002/dys.1505

Lallier, M., Tainturier, M. J., Dering, B., Donnadieu, S., Valdois, S., \& Thierry, G. (2010). Behavioral and ERP evidence for amodal sluggish attentional shifting in developmental dyslexia. Neuropsychologia, 48(14), 4125-4135. doi:https://doi.org/10. 1016/j.neuropsychologia.2010.09.027

Lallier, M., Thierry, G., Tainturier, M. J., Donnadieu, S., Peyrin, C., Billard, C., \& Valdois, S. (2009). Auditory and visual stream segregation in children and adults: An assessment of the amodality assumption of the "sluggish attentional shifting" theory of dyslexia. Brain Research, 1302, 132-147. doi:https://doi.org/10.1016/j. brainres.2009.07.037

Livingstone, M. S., \& Hubel, D. H. (1987). Psychophysical evidence for separate channels for the perception of form, color, movement, and depth. Journal of Neuroscience, 7(11), 3416-3468.

Livingstone, M. S., Rosen, G. D., Drislane, F. W., \& Galaburda, A. M. (1991). Physiological and anatomical evidence for a magnocellular defect in developmental dyslexia. Proceedings of the National Academy of Sciences of the United States of America, 88(18), 7943-7947. 
Lu, T., Liang, L., \& Wang, X. (2001). Temporal and rate representations of time-varying signals in the auditory cortex of awake primates. Nature Neuroscience, 4(11), 1131-1138. doi:https://doi.org/10. $1038 / \mathrm{nn} 737$

Mascheretti, S., Gori, S., Trezzi, V., Ruffino, M., Facoetti, A., \& Marino, C. (2018). Visual motion and rapid auditory processing are solid endophenotypes of developmental dyslexia. Genes, Brain and Behavior, 17(1), 70-81. doi:https://doi.org/10.1111/gbb.12409

Maunsell, J. H. R., \& Newsome, W. T. (1987). Visual processing in monkey extrastriate cortex. Annual Review of Neuroscience, 10(1), 363-401.

McLean, G. M. T., Stuart, G. W., Coltheart, V., \& Castles, A. (2011). Visual temporal processing in dyslexia and the magnocellular deficit theory: The need for speed? Journal of Experimental Psychology: Human Perception and Performance, 37(6), 1957-1975. doi:https:// doi.org/10.1037/a0024668

Menghini, D., Finzi, A., Benassi, M., Bolzani, R., Facoetti, A., Giovagnoli, S., ... Vicari, S. (2010). Different underlying neurocognitive deficits in developmental dyslexia: A comparative study. Neuropsychologia, 48(4), 863-872. doi:https://doi.org/10. 1016/j.neuropsychologia.2009.11.003

Morrone, M. C., Tosetti, M., Montanaro, D., Fiorentini, A., Cioni, G., \& Burr, D. C. (2000). A cortical area that responds specifically to optic flow, revealed by fMRI. Nature Neuroscience, 3(12), 1322-1328. doi:https://doi.org/10.1038/81860

Pammer, K. (2014). Temporal sampling in vision and the implications for dyslexia. Frontiers in Human Neuroscience, 7, 933. doi:https://doi. org/10.3389/fnhum.2013.00933

Pokorny, J. (2011). Review: Steady and pulsed pedestals, the how and why of post-receptoral pathway separation. Journal of Vision, 11(5), 7-7. doi:https://doi.org/10.1167/11.5.7

Pöppel, E. (1997). A hierarchical model of temporal perception. Trends in Cognitive Sciences, 1(2), 56-61. doi:https://doi.org/10.1016/S13646613(97)01008-5

Pöppel, E. (2009). Pre-semantically defined temporal windows for cognitive processing. Philosophical Transactions of the Royal Society B: Biological Sciences, 364(1525), 1887-1896. doi:https://doi.org/ 10.1098/rstb.2009.0015

Ramus, F., \& Ahissar, M. (2012). Developmental dyslexia: The difficulties of interpreting poor performance, and the importance of normal performance. Cognitive Neuropsychology, 29(1/2), 104-122. doi: https://doi.org/10.1080/02643294.2012.677420

Raven, J. (1998). Manual for Raven's Advanced Progressive Matrices. Oxford, England: Oxford Psychologists Press.

Roach, N. W., \& Hogben, J. H. (2007). Impaired filtering of behaviourally irrelevant visual information in dyslexia. Brain, 130(3), 771-785.

Roncon, L., Busch, N. A., \& Melcher, D. (2018). Alpha-band sensory entrainment alters the duration of temporal windows in visual perception. Scientific Reports, 8(1), 11810. doi:https://doi.org/10.1038/ s41598-018-29671-5

Ronconi, L., \& Melcher, D. (2017). The role of oscillatory phase in determining the temporal organization of perception: Evidence from sensory entrainment. The Journal of Neuroscience, 37(44), 10636 10644. doi:https://doi.org/10.1523/JNEUROSCI.1704-17.2017

Ronconi, L., Oosterhof, N. N., Bonmassar, C., \& Melcher, D. (2017). Multiple oscillatory rhythms determine the temporal organization of perception. Proceedings of the National Academy of Sciences of the United States of America, 114(51), 13435-13440. doi:https://doi. org/10.1073/pnas.1714522114

Ronconi, L., Pincham, H. L., Cristoforetti, G., Facoetti, A., \& Szucs, D. (2016a). Shaping prestimulus neural activity with auditory rhythmic stimulation improves the temporal allocation of attention. NeuroReport, 27(7), 487-494. doi:https://doi.org/10.1097/WNR. 0000000000000565
Ronconi, L., Pincham, H. L., Szucs, D., \& Facoetti, A. (2016b). Inducing attention not to blink: auditory entrainment improves conscious visual processing. Psychological Research, 80(5), 774-784. doi: https://doi.org/10.1007/s00426-015-0691-8

Samaha, J., Bauer, P., Cimaroli, S., \& Postle, B. R. (2015). Top-down control of the phase of alpha-band oscillations as a mechanism for temporal prediction. Proceedings of the National Academy of Sciences of the United States of America, 112(27), 8439-8444. doi:https://doi.org/10.1073/pnas.1503686112

Samaha, J., \& Postle, B. R. (2015). The Speed of Alpha-Band Oscillations Predicts the Temporal Resolution of Visual Perception. Current Biology, 25(22), 2985-2990. doi:https://doi. org/10.1016/j.cub.2015.10.007

Sartori, G., Job, R., \& Tressoldi, P. E. (1995). Batteria per la valutazione della dislessia e della disortografia evolutiva [Battery for the assessment of dyslexia and developmental dysorthography]. Florence, Italy: Organizzazioni Speciali.

Sharp, P., Melcher, D., \& Hickey, C. (2018). Endogenous attention modulates the temporal window of integration. Attention, Perception, \& Psychophysics, 1-15. doi:https://doi.org/10.3758/s13414-0181506-y

Sharp, P., Melcher, D., \& Hickey, C. (2019). Different effects of spatial and temporal attention on the integration and segregation of stimuli in time. Attention, Perception, \& Psychophysics, 81(2), 433-441. doi:https://doi.org/10.3758/s13414-018-1623-7

Shaywitz, B. A., Shaywitz, S. E., Blachman, B. A., Pugh, K. R., Fulbright, R. K., Skudlarski, P., ... Gore, J. C. (2004). Development of left occipitotemporal systems for skilled reading in children after a phonologically- based intervention. Biological Psychiatry, 55(9), 926-933. doi:https://doi.org/10.1016/J. BIOPSYCH.2003.12.019

Stanley, G., \& Hall, R. (1973). Short-term visual information processing in dyslexics. Child Development, 44(4), 841. doi:https://doi.org/10. $2307 / 1127735$

Stein, J. (2019). The current status of the magnocellular theory of developmental dyslexia. Neuropsychologia, 130, 66-77. doi:https://doi. org/10.1016/j.neuropsychologia.2018.03.022

Stein, J., \& Talcott, J. (1999). Impaired neuronal timing in developmental dyslexia - the magnocellular hypothesis. Dyslexia , 5(2), 59-77. doi: https://doi.org/10.1002/(SICI)1099-0909(199906)5:2<59::AIDDYS134>3.0.CO;2-F

Stein, J., \& Walsh, V. (1997). To see but not to read; the magnocellular theory of dyslexia. Trends in Neurosciences, 20(4), 147-152.

Tallal, P. (1980). Auditory temporal perception, phonics, and reading disabilities in children. Brain and Language, 9(2), 182-198. doi: https://doi.org/10.1016/0093-934X(80)90139-X

Tallal, P. (2004). Improving language and literacy is a matter of time. Nature Reviews Neuroscience, 5(9), 721-728.

van Wassenhove, V. (2016). Temporal cognition and neural oscillations. Current Opinion in Behavioral Sciences, 8, 124-130. doi:https:// doi.org/10.1016/J.COBEHA.2016.02.012

VanRullen, R. (2016). Perceptual cycles. Trends in Cognitive Sciences, $20(10), 723-735$.

Vidyasagar, T. R. (2013). Reading into neuronal oscillations in the visual system: Implications for developmental dyslexia. Frontiers in Human Neuroscience, 7, 811. doi:https://doi.org/10.3389/fnhum. 2013.00811

Vidyasagar, T. R. (2019). Visual attention and neural oscillations in reading and dyslexia: Are they possible targets for remediation? Neuropsychologia, 130, 59-65. doi:https://doi.org/10.1016/J. NEUROPSYCHOLOGIA.2019.02.009

Vidyasagar, T. R., \& Pammer, K. (1999). Impaired visual search in dyslexia relates to the role of the magnocellular pathway in attention. NeuroReport, 10(6), 1283-1287.

Vidyasagar, T. R., \& Pammer, K. (2010). Dyslexia: a deficit in visuospatial attention, not in phonological processing. Trends in 
Cognitive Sciences, 14(2), 57-63. doi:https://doi.org/10.1016/J. TICS.2009.12.003

Visser, T. A. W., Boden, C., \& Giaschi, D. E. (2004). Children with dyslexia: Evidence for visual attention deficits in perception of rapid sequences of objects. Vision Research, 44(21), 2521-2535.

Wutz, A., \& Melcher, D. (2014). The temporal window of individuation limits visual capacity. Frontiers in Psychology, 5, 952. doi:https:// doi.org/10.3389/fpsyg.2014.00952

Wutz, A., Weisz, N., Braun, C., \& Melcher, D. (2014). Temporal windows in visual processing: "Prestimulus brain state" and "poststimulus phase reset" segregate visual transients on different temporal scales. The Journal of Neuroscience, 34(4), 1554-1565. doi:https:// doi.org/10.1523/JNEUROSCI.3187-13.2014

Wutz, A., Melcher, D., \& Samaha, J. (2018). Frequency modulation of neural oscillations according to visual task demands. Proceedings of the National Academy of Sciences, 115(6), 1346-1351. doi:https:// doi.org/10.1073/pnas.1713318115

Wutz, A., Muschter, E., van Koningsbruggen, M. G., Weisz, N., \& Melcher, D. (2016). Temporal integration windows in neural processing and perception aligned to saccadic eye movements. Current Biology, 26, 1-10.

Zion Golumbic, E. M., Poeppel, D., \& Schroeder, C. E. (2012). Temporal context in speech processing and attentional stream selection: A behavioral and neural perspective. Brain and Language, 122(3), 151-161. doi:https://doi.org/10.1016/j.bandl.2011.12.010

Publisher's note Springer Nature remains neutral with regard to jurisdictional claims in published maps and institutional affiliations. 